\title{
Research on Risk Assessment and Control of Real Estate Project Loan in China
}

\author{
Yi Liu ${ }^{\mathrm{a}}$, Meiyan Yang ${ }^{\mathrm{b}}$ and Siran Wang ${ }^{\mathrm{c}}$ \\ Business School, University of Jinan, P.R.China \\ a921089327@qq.com, ${ }^{b} 634500456 @ q q . c o m,{ }^{c} 731208868 @ q q . c o m$
}

Keywords: Real estate projects, Bank loans, Risk management

\begin{abstract}
Real estate development funds rely too much on bank loans, making the real estate investment market risk and financing credit risk concentrated in the bank. Based on the analysis of the loan risk factors in the real estate project construction, this paper establishes the risk assessment index system of the real estate project loan, and constructs the risk evaluation model of commercial bank's real estate loan, ultimately puts forward the measures to prevent the project risk control through the risk assessment.
\end{abstract}

\section{Introduction}

China 's real estate has a really rapid pace of development since the real estate reform, real estate projects have become an important way to promote economic development. Large-scale real estate investment improved national living conditions, increased the national happiness index and brought great benefits. At the same time, real estate developers are in pursuit of high profits and cost savings; the risk awareness is weak, and risk management is not perfect. Blind investment and development lead to many houses hoarding without sales and bring losses to the real estate manufacturers, simultaneously endangering China's real estate industry[1]. Therefore, it requires a comprehensive assessment of the investment risk of real estate projects to determine the degree of investment risk, to develop a real estate investment management system, to reduce investment risk, and to put forward measures to maintain the healthy development of the real estate industry.

\section{Literature Review}

China's project risk management research began in the 1980s, when most of the enterprises learned from foreign advanced experience, and they began to sum up experience in the practice slowly from the company's development projects, and gradually they have established suitable theory and methods for project risk management of Chinese enterprises. After 2000, Chinese scholars have reached a new height on the research of project risk management on the basis of advanced foreign research. Guo Jiqiu and Tang Huizhe (2005) put forward the risk management of each construction link according to the risk management stage and each stage of the risk management was analyzed theoretically. Bi-zhou, Zhai Li (2006) summarized a lot of methods, techniques, principle problems of the project risk management and published the book, which is a monument on China's project risk management research. As for the research of risk assessment of real estate project, Chen Shanshou(2010) established risk management index system, then made a comprehensive evaluation of the real estate project system and got the risk management score; finally he put forward the reform of real estate project risk management. Li Chenzhen, Xu Dongsheng(2014)) studied the dynamic control method of the project and used advanced network planning technology to calculate the project model, which made the birth of the quantitative project risk management and the simulation model was used to simulate the project.

It can be seen that the scholars have some research on the method of project risk management and put forward some innovative analysis methods. However, many risk management methods are not scientific and have not been tested in practice, it need to further use the practice to test the theory and accordingly improve the new methods and theories. 


\section{Analysis on the Influencing Factors of Loan Risk of Real Estate Development Project}

Political Environmental Risk. In recent years, the state introduced a lot of policies and regulations in order to save the market, such as financial institutions lending rates decrease, provident fund loans down payment decline, government purchase incentives, these measures have increased the demand for real estate, while bringing real estate project loan risk.

Internal Business Risk. As the changes of capital supply and demand market and the macroeconomic environment, the construction funds fail to implement and put in place, resulting in the project can not be implemented on schedule, which increase financing risk. When a certain amount of funds are put into real estate projects, investment risk may generate due to demand changes in market and the impact of the final income and expected return[2]. Hysteresis of enterprise financial movement, which is caused by the impact of uncertainty factors in supply, production, sales of all aspects in the production and management process, may easily lead to operational risks.

Credit Risk. With the market supply increased, real estate development investment continued to increase in 2012. The risk prevention mechanism should be improved, and corporate responsibility and behavior should be standardized to avoid the situation in which the risk is the bank to bear. The state should strengthen market regulation, curb housing prices rose too fast, strengthen credit management to avoid real estate credit risk.

Construction Risk. With the market supply increased, real estate development investment continued to increase in 2012. The risk prevention mechanism should be improved, and corporate responsibility and behavior should be standardized to avoid the situation in which the risk is the bank to bear. The state should strengthen market regulation, curb housing prices rose too fast, strengthen credit management to avoid real estate credit risk.

\section{Loan Risk Assessment Index System of Real Estate Development Project}

Design Principles of Loan Risk Evaluation Index of Real Estate Development Project. When we design an evaluation index system, first we should have scientific theory to be a guide, so that the evaluation index system can be rigorous and reasonable in the basic concepts and logical structure, and seize the essence of the evaluation object, at the same time, it should be targeted. The evaluation object must be measured by a number of indicators, which are interrelated and mutually constrained[3]. Some indicators have a horizontal link, reflecting the different side of the mutual restraint relationship. Meanwhile, the boundaries of indicators within the same level should be clear as far as possible, avoiding mutual contact with each other, and reflecting a strong system.

Selection of Evaluation Indicators. Through the analysis of the actual situation of the project, making a summary of all aspects of the risk that the project will face and monitoring the risk changes closely, then taking transfer or mitigation measures when the risk occurs. This system includes the target layer, the main factor layer and the sub-factor layer, as shown in Table 1.

Table 1 Project Investment Risk Index System

\begin{tabular}{ccc}
\hline Target layer & Main factor layer & Sub-factor layer \\
\hline & External risk & Government financial ability risk \\
Project & & Government public policy risk \\
Investment & & Cash flow risk \\
Risk & Internal risk & Financing efficiency risk \\
Analysis & & Interest rate risk \\
& & Cost overrun risk \\
& Construction risk & Project quality risk \\
& & Project progress risk \\
\hline
\end{tabular}




\section{Loan Risk Comprehensive Evaluation of Real Estate Development Project}

Based on the established loan risk index system of real estate project, the fuzzy evaluation model is used to evaluate the loan risk of "Jinyu Runyuan" housing development project. The loan of the project is intended to be secured by collateral, the collateral is effective and the liquidity is strong, also the company's land and construction in progress are set up to meet the guarantee conditions and have the guarantee capability.

The Construction of the Commentary. In the loan risk comprehensive evaluation of "Jinyu Runyuan" housing development project, ${ }{ }_{P}$ means the risk level, and the risk level is divided into large, relatively large, medium, relatively small and very small these five grades.

Weight Assignment Determination Between the Index Layers. Weight assignment determination need several experts to do this distribution. Through the weight evaluation, the matrix is obtained, the weight value form is changed from decimal to integer due to writing convenience, and we get the following matrix:

$$
W_{h i}=\left[\begin{array}{cccccccccccc}
5 & 15 & 5 & 10 & 5 & 10 & 5 & 10 & 10 & 10 & 10 & 5 \\
5 & 15 & 5 & 15 & 5 & 10 & 5 & 5 & 10 & 15 & 5 & 5 \\
5 & 20 & 10 & 5 & 5 & 5 & 10 & 15 & 5 & 10 & 5 & 5 \\
10 & 10 & 10 & 5 & 5 & 10 & 5 & 15 & 10 & 5 & 10 & 5 \\
5 & 15 & 5 & 10 & 5 & 5 & 15 & 5 & 10 & 15 & 5 & 5 \\
5 & 15 & 5 & 10 & 5 & 5 & 15 & 5 & 10 & 15 & 5 & 5 \\
5 & 10 & 10 & 15 & 5 & 5 & 5 & 10 & 5 & 15 & 5 & 10 \\
5 & 10 & 5 & 10 & 10 & 5 & 10 & 10 & 5 & 15 & 10 & 5 \\
5 & 10 & 10 & 5 & 10 & 10 & 5 & 10 & 10 & 10 & 10 & 5 \\
5 & 15 & 5 & 5 & 5 & 10 & 5 & 15 & 5 & 15 & 10 & 5
\end{array}\right] / 100
$$

The similarity matrix $\left(R_{h g}\right)_{10 \times 10}$ is got by similarity coefficient formula

$$
\begin{aligned}
R_{h g}= & 1-\left(\frac{1}{n} \sum_{i=1}^{m}\left(W_{h i}-W_{g i}\right)^{2}\right)^{\frac{1}{2}}: \\
R_{h g} & =\left[\begin{array}{ccccccccccc}
1 & 0.965 & 0.965 & 0.959 & 0.971 & 0.959 & 0.965 & 0.971 & 0.965 & 0.965 \\
0.965 & 1 & 0.946 & 0.942 & 0.965 & 0.965 & 0.971 & 0.959 & 0.954 & 0.954 \\
0.965 & 0.946 & 1 & 0.954 & 0.954 & 0.950 & 0.954 & 0.954 & 0.954 & 0.965 \\
0.959 & 0.942 & 0.954 & 1 & 0.939 & 0.946 & 0.959 & 0.950 & 0.971 & 0.959 \\
0.971 & 0.965 & 0.954 & 0.939 & 1 & 0.954 & 0.959 & 0.965 & 0.950 & 0.950 \\
0.959 & 0.965 & 0.950 & 0.946 & 0.954 & 1 & 0.959 & 0.965 & 0.954 & 0.954 \\
0.965 & 0.971 & 0.954 & 0.959 & 0.959 & 0.959 & 1 & 0.954 & 0.965 & 0.954 \\
0.971 & 0.959 & 0.954 & 0.950 & 0.965 & 0.965 & 0.954 & 1 & 0.965 & 0.965 \\
0.965 & 0.954 & 0.954 & 0.971 & 0.950 & 0.954 & 0.965 & 0.965 & 1 & 0.965 \\
0.965 & 0.954 & 0.965 & 0.959 & 0.950 & 0.954 & 0.954 & 0.965 & 0.965 & 1
\end{array}\right]
\end{aligned}
$$

By the degree of deviation $P_{h}=\sum R_{h g}$, we got

$$
P=\left(p_{1}, p_{2}, \ldots, p_{10}\right)^{T}=(9.685,9.621,9.596,9.579,9.607,9.606,9.640,9.648,9.643,9.631)^{T} \text {, }
$$

By the deviation coefficient $D_{h}=\frac{p_{\max }-p_{h}}{p_{\max }} \times 100 \%$, we got

$$
D=\left(D_{1}, D_{2}, \ldots, D_{10}\right)=(0,0.66 \%, 0.91 \%, 1.09 \%, 0.81 \%, 0.82 \%, 0.46 \%, 0.38 \%, 0.43 \%, 0.56 \%) \text {. }
$$


Set the limit $D_{0}=0.9 \%$, so we can see the weight values of experts 5 and 6 are void, so we determine the weight value according to other eight experts. The weight of the risk factors is obtained by normalizing the degree of correlation. Thus, the weight matrices of $U_{2}, U_{3}, U_{4}, U_{5}$ are as follows:

$$
\begin{aligned}
& A_{2}=\{0.656,0.344\}, A_{3}=\{0.529,0.471\}, A_{4}=\{0.296,0.308,0.397\}, A_{6}=\{0.573,0.427\} . \\
& \text { The weight matrix of } U \text { is } A=\{0.055,0.221,0.155,0.247,0.076,0.185,0.061\}
\end{aligned}
$$

Fuzzy Comprehensive Evaluation. First, we established a fuzzy evaluation matrix:

$$
\mathrm{R}=\left[\begin{array}{cccc}
0.3 & 0.4 & 0.2 & 0.1 \\
0.2 & 0.4 & 0.3 & 0.1 \\
0.5 & 0.3 & 0.2 & 0.1 \\
0.4 & 0.3 & 0.2 & 0.1 \\
0.1 & 0.2 & 0.4 & 0.3 \\
0.3 & 0.2 & 0.1 & 0.4 \\
0.2 & 0.2 & 0.3 & 0.3 \\
0.5 & 0.2 & 0.3 & 0
\end{array}\right]
$$

From the formula $\mathrm{Bi}=\mathrm{Ai} \cdot \mathrm{Ri}$, we got the evaluation membership matrix:

$$
\mathrm{B}=\left[\begin{array}{llll}
0.345 & 0.360 & 0.235 & 0.060 \\
0.265 & 0.235 & 0.240 & 0.260 \\
0.380 & 0.200 & 0.300 & 0.120
\end{array}\right]
$$

From the formula $B{ }^{\prime}=A \cdot B$, the fuzzy comprehensive evaluation set is:

$$
B^{\prime}=\left[\begin{array}{llll}
0.3235 & 0.2620 & 0.2565 & 0.1580
\end{array}\right]
$$

Evaluation Results and Analysis. According to the results of fuzzy comprehensive evaluation,

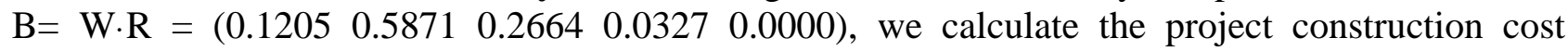
management index score of 0.5871 , the risk management evaluation is in a good level, the score of each sub-target evaluation is also good, the risk management system evaluation result level is good; expected results of the risk management system evaluation rating is also good.

\section{Conclusion and Suggestion}

Focus on the Feasibility Study of Real Estate Development Projects. The feasibility study is to determine the decisive work before the construction project, and it is a scientific demonstration of a comprehensive technical and economic analysis of the proposed project prior to the investment decision. In the investment management, the feasibility study refers to the research, analysis and comparison of social, economic, technical and other factors, and forecast the social and economic benefits after completion. On the basis of this, the necessity and feasibility of project construction, the profitability of finance, the rationality of economy, the advancedness and adaptability of technology and the conditions of construction are put forward, so as to provide scientific basis for investment decision.

Enhance the Level of Risk Management of Real Estate Project Development. Real estate development must comply with the various laws and regulations of construction industry; it should be in strict accordance with the law construction. In the personnel system of the projects, we must establish a very clear responsibility system, and the problem must be solved when occurs, also, the implementation of punishment and incentives are necessary to increase the enthusiasm of the staff work. At the same time, a reasonable system may be established to coordinate the work between the various departments, so as to increase efficiency, avoid unnecessary problems of construction accidents[4]. In addition, the project can increase investment in people, finances, materials and technology, and strengthen the technical training and education of personnel, improve the technical level and safety awareness of workers, then they would be familiar with the latest technology and methods to ensure scientific construction. When there are significant technology or engineering 
design changes, management staff should be organized to hold a consultation meeting to jointly study the solution, furthermore the establishment of a good internal system is to avoid the problem that information is not circulated.

\section{Acknowledgements}

This paper obtained the funding of the National Social Science Fund Project Transformation and Upgrading of China's Manufacturing Industry Coupled with Rising Labor Costs Research (project approval No: 16BGL098);Environmental Audit of Ecological Civilization Construction (project approval No: 15AGL015); Shandong Province Social Science Planning Project Evaluation and Countermeasures on Ecological Civilization in Shandong Province Construction Performance (project approval No: 15BKJJ04) .

\section{References}

[1] Y.J. Wang and X.J. Wang. Commercial bank credit moral hazard analysis and preventive measures [J]. Journal of Wuhan University of Technology, 2009, (12): 145-147.(In Chinese)

[2] S.B. Bai. Study on Early Warning of Financing Risk of Enterprise Supply Chain Based on Ordered Logistic Model [J]. Economic Science and Technology, 2010,(6): 66-71.(In Chinese)

[3] L.Xu. Research of Small and medium-sized real estate enterprises credit risk assessment of loans [D]. Southwest University of Finance and Economics, 2011: 22-41.(In Chinese)

[4] Y. Zhu. Research of Macro-control under the real estate development loan risk management research [D]. Southwest University of Finance and Economics, 2011: 1-42.(In Chinese) 